\title{
Wicking-Driven Evaporation Self-Assembly of Carbon Nanotubes on Fabrics: Generating Controlled Orientational Structures
}

Chenglong Li,,$^{\dagger}$ Chuntao Lan, ${ }^{\dagger}$ Min Guo, ${ }^{\dagger}$ Ni Wang, ${ }^{\dagger}$ and Ying Ma*,+,

†Key Laboratory of Textile Science \& Technology of Ministry of Education, College of Textiles, Donghua University, Shanghai 201620, China

\#nnovation Center for Textile Science and Technology, Donghua University,

Shanghai 200051, China

*E-mail: yingma@dhu.edu.cn

Number of pages: 6

Number of figures: 8

Number of tables: 1

Figure S1. Deposition mass (g) of the CNT films on per unit cotton fabric (g) for the sample assembled at different concentrations of alginate.

Figure S2. Sheet resistance of the CNT fabrics assembled at different concentrations of alginate.

Figure S3. XPS analysis of the (CNT)*5 films assembled at different concentrations of alginate.

Figure S4. CNT content (g) on per unit cotton fabric (g) for the (CNT)*5 films assembled at different concentrations of alginate.

Figure S5. Deposition mass (g) of the CNT films on per unit cotton fabric (g) for the sample assembled at different $\mathrm{pH}$.

Figure S6. Sheet resistance of the CNT fabrics assembled at different $\mathrm{pH}$.

Figure S7. XPS analysis of the (CNT)*5 films assembled at different $\mathrm{pH}$.

Figure S8. CNT content (g) on per unit cotton fabric (g) for the (CNT)*5 films assembled at different $\mathrm{pH}$.

Table S1. Viscosity of the CNT suspensions with different concentrations of alginate. 


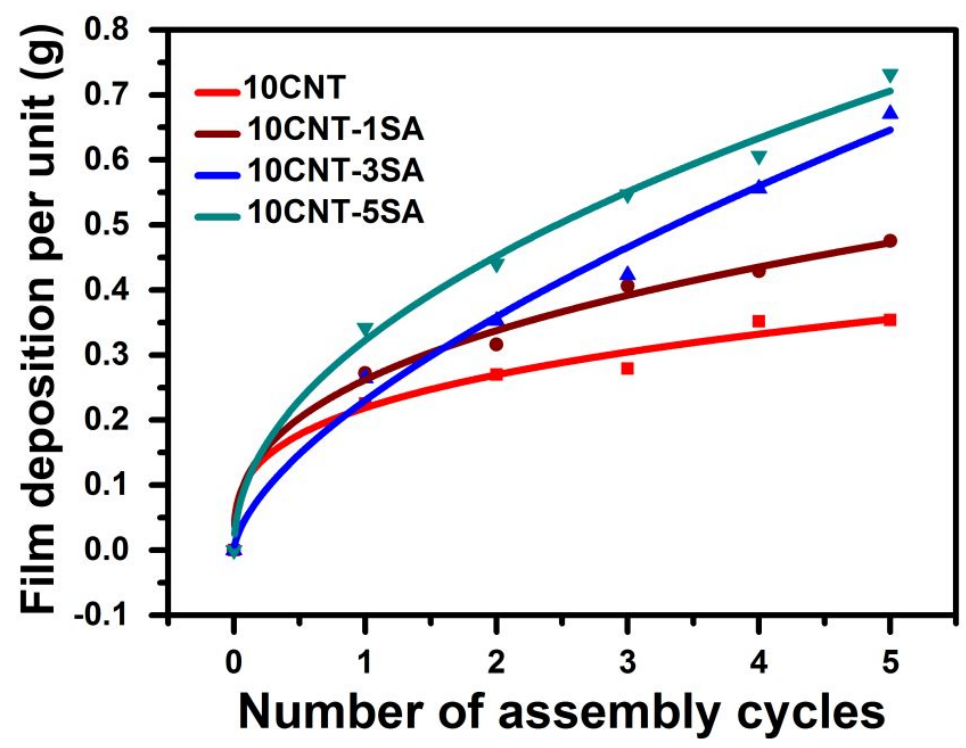

Figure S1. Deposition mass (g) of the CNT films on per unit cotton fabric (g) for the sample assembled at different concentrations of alginate.

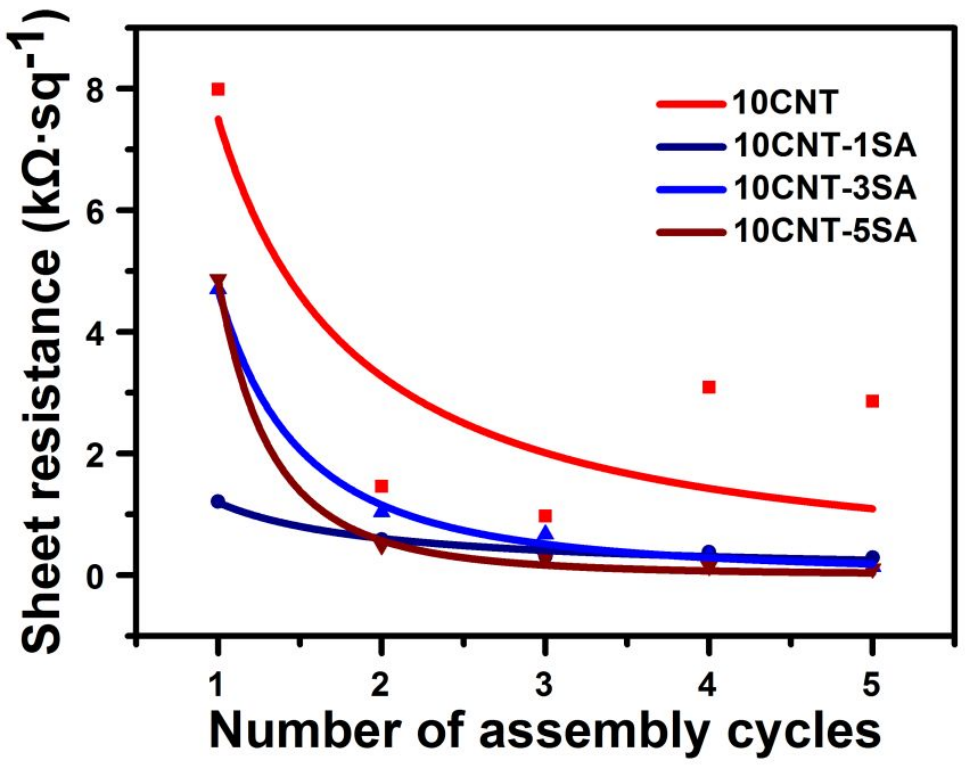

Figure S2. Sheet resistance of the CNT fabrics assembled at different concentrations of alginate. 


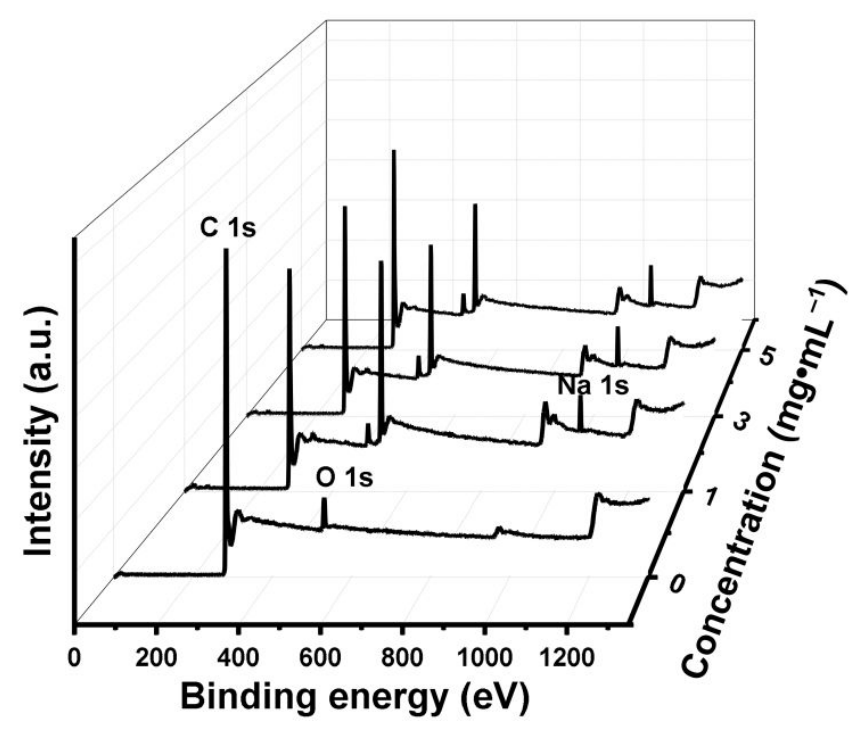

Figure S3. XPS analysis of the (CNT)*5 films assembled at different concentrations of alginate.

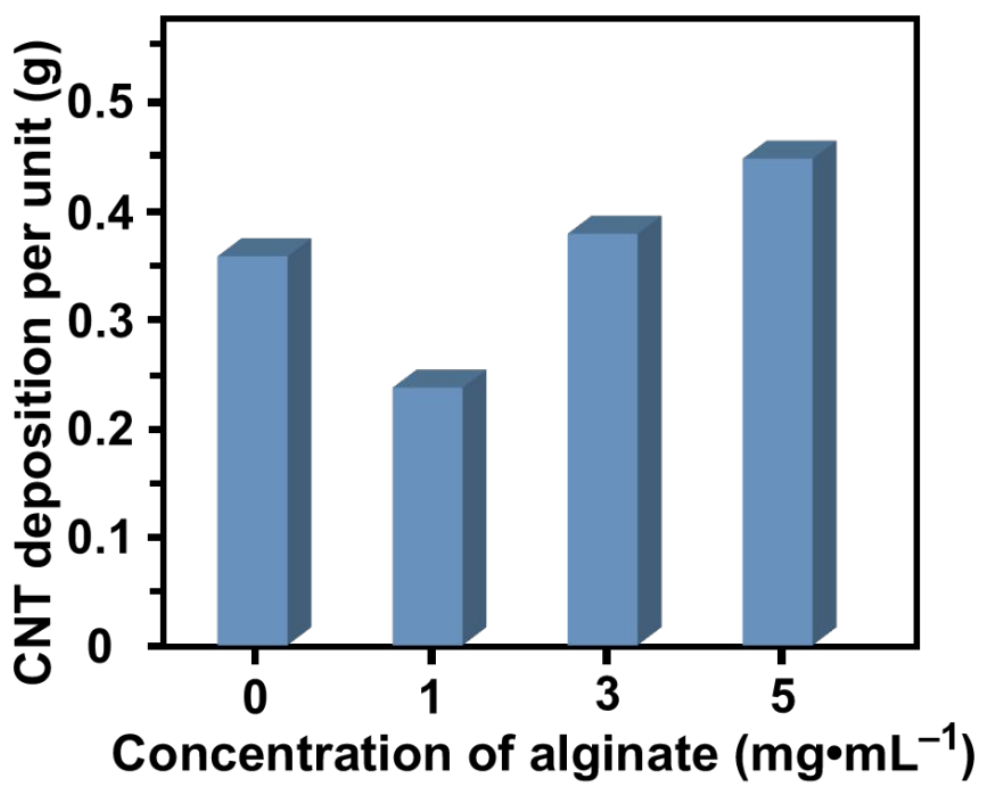

Figure S4. CNT content $(\mathrm{g})$ on per unit cotton fabric $(\mathrm{g})$ for the $(\mathrm{CNT}) * 5$ films assembled at different concentrations of alginate. 


\begin{tabular}{cc} 
alginate concentration $\left(\mathrm{mg} \cdot \mathrm{mL}^{-1}\right)$ & Viscosity $(\mathrm{cP})$ \\
\hline 0 & 4.07 \\
1 & 8.16 \\
3 & 18.2 \\
5 & 31.98 \\
\hline
\end{tabular}

Table S1. Viscosity of the CNT suspensions with different concentrations of alginate.

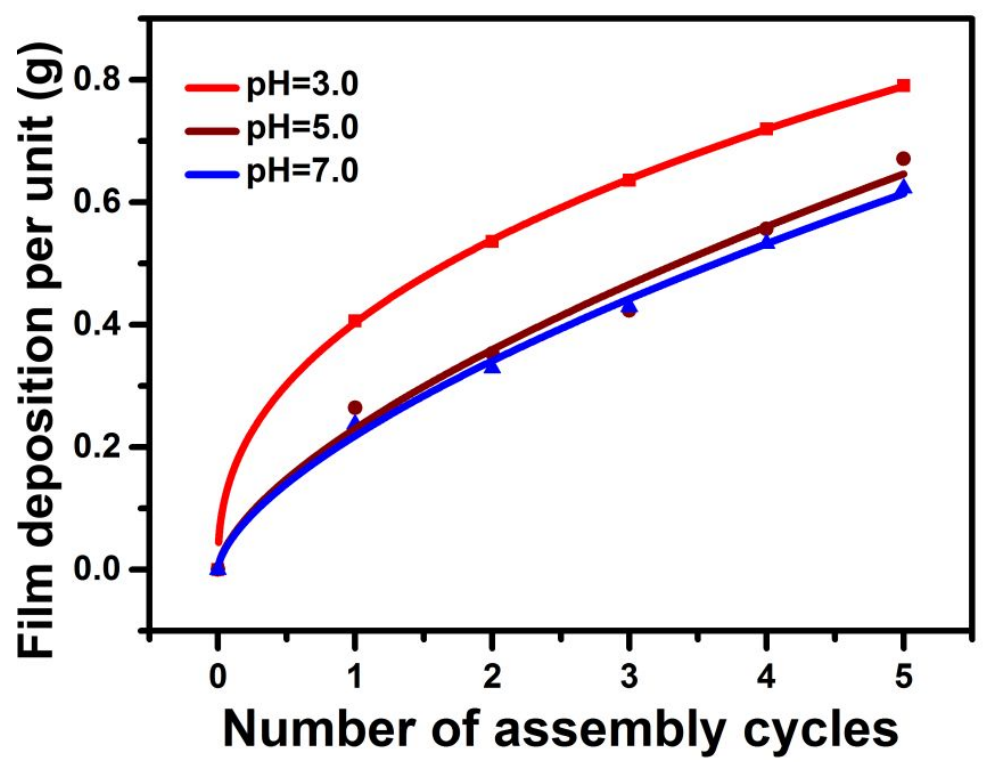

Figure S5. Deposition mass (g) of the CNT films on per unit cotton fabric (g) for the sample assembled at different $\mathrm{pH}$. 


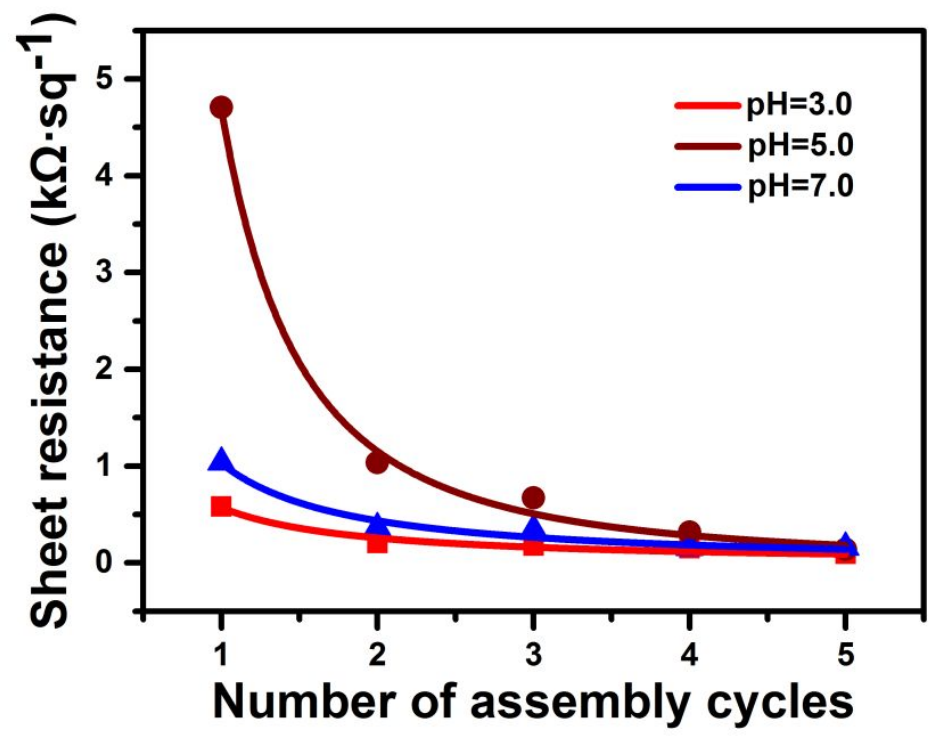

Figure S6. Sheet resistance of the CNT fabrics assembled at different $\mathrm{pH}$.

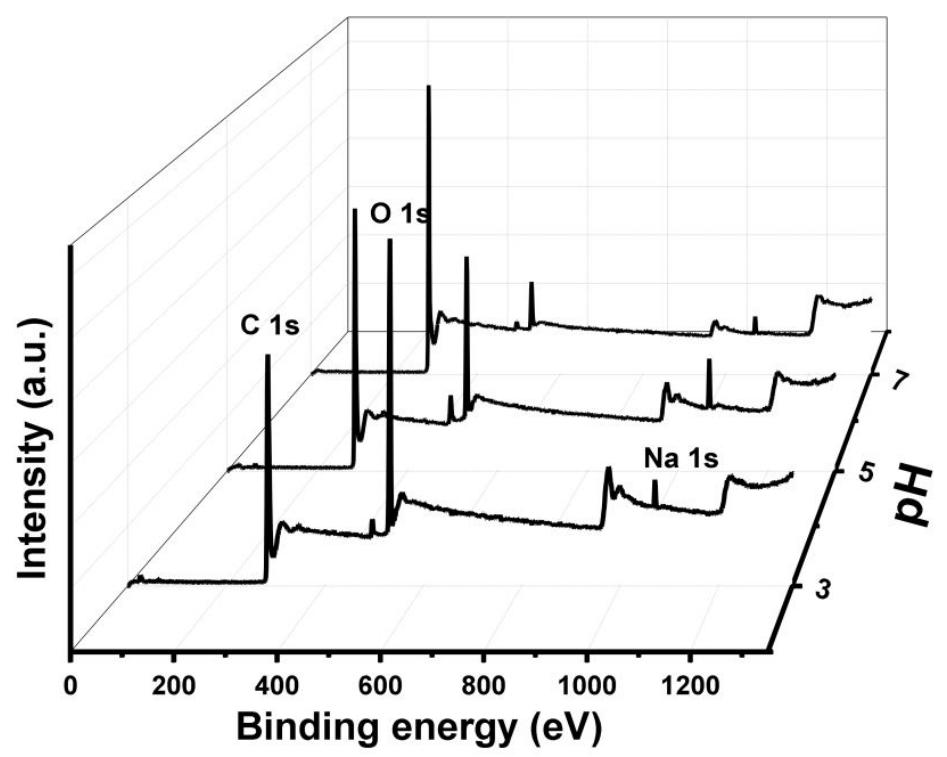

Figure S7. XPS analysis of the (CNT)*5 films assembled at different $\mathrm{pH}$. 


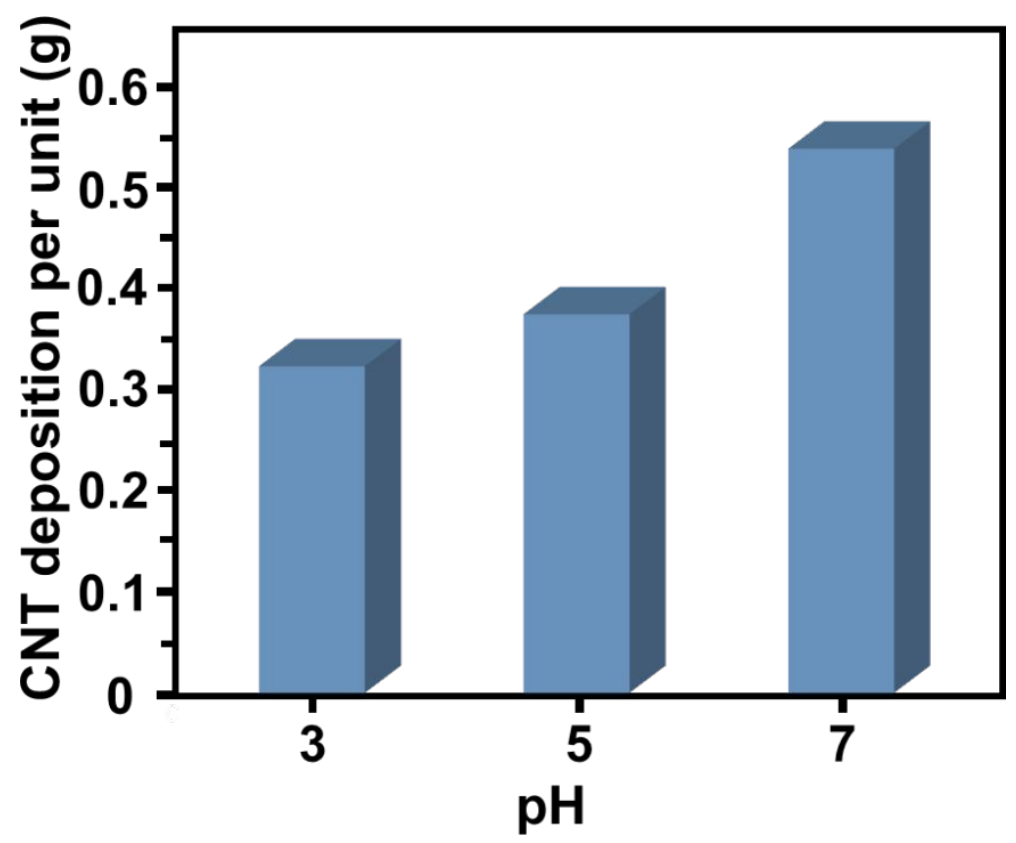

Figure S8. CNT content $(\mathrm{g})$ on per unit cotton fabric $(\mathrm{g})$ for the $(\mathrm{CNT}) * 5$ films assembled at different $\mathrm{pH}$. 